$\begin{array}{ll}\text { le portiQue } & \text { Le Portique } \\ \text { Revue de philosophie et de sciences humaines }\end{array}$

16 | 2005

Sartre. Conscience et liberté

\title{
La morale de Sartre . Une reconstruction
}

\section{Gerhard Seel}

\section{OpenEdition}

\section{Journals}

Édition électronique

URL : http://journals.openedition.org/leportique/737

DOI : 10.4000/leportique.737

ISSN : $1777-5280$

\section{Éditeur}

Association "Les Amis du Portique"

Édition imprimée

Date de publication : 1 septembre 2005

ISSN : 1283-8594

\section{Référence électronique}

Gerhard Seel, «La morale de Sartre. Une reconstruction », Le Portique [En ligne], 16 | 2005, mis en ligne le 15 juin 2008, consulté le 25 mars 2021. URL : http://journals.openedition.org/leportique/737 ; DOI : https://doi.org/10.4000/leportique.737

Ce document a été généré automatiquement le 25 mars 2021

Tous droits réservés 


\title{
La morale de Sartre. Une reconstruction
}

\author{
Gerhard Seel
}

Introduction

Dans son premier ouvrage, en 1936, Sartre avait déjà esquissé le programme d'une éthique : « Il n'est pas nécessaire [...] que l'objet précède le sujet pour que les pseudovaleurs spirituelles s'évanouissent et pour que la morale retrouve ses bases dans la réalité » (TrE, p. 86). En 1943, à la fin de L'Être et le Néant, Sartre avait annoncé que son prochain ouvrage porterait sur l'éthique. Cependant, Sartre n'a jamais publié de philosophie morale ${ }^{1}$. Alain Renaut tente d'expliquer ce fait par une déficience interne de la philosophie morale de Sartre, par un échec théorique, et il explique cet échec par le fait que Sartre n'est jamais parvenu à « concevoir à quel point les deux moments qui définissent l'humanisme authentique (affirmer que le propre de l'homme est le néant, mais aussi soutenir qu'il peut viser l'universel) sont inséparables ${ }^{2}{ }^{2}$. Cependant une analyse minutieuse du concept sartrien de la valeur ${ }^{3}$ peut montrer que celui-ci implique justement la thèse que l'homme non seulement est susceptible de viser l'universel, mais encore ne cesse de le faire. Dans cet article, nous voulons examiner si cette constatation vaut également dans le domaine de la morale. Nous espérons ainsi montrer que l'humanisme de Sartre ne se limite pas à des déclarations et à un engagement politique sérieux et courageux (ce qui n'est pas à mépriser), mais trouve également un fondement argumenté dans sa philosophie pratique. Ce faisant, nous mettrons aussi en lumière les points de sa pensée morale qui demeurent problématiques et discutables.

2 Dans cette entreprise, il faut tenir compte du fait que nous n'avons pas affaire chez Sartre à une, mais à trois morales, qui divergent sur des points importants. C'est Sartre lui-même qui distingue, dans ses entretiens avec André Gorz et Jean Pouillon ${ }^{4}$, mais aussi dans un entretien accordé à Michel Sicard (1977-1978) ${ }^{5}$, trois phases dans le développement de sa philosophie morale.

3 La première est la phase de L'Être et le Néant, de L'existentialisme est un humanisme (1946) et des Cahiers pour une morale (1947-1948). Tout ceci constitue ce que Sartre appellera 
plus tard «La Morale » ou aussi, peut-être en parodiant Aristote, « La grande morale ». Mais, bien plus que ces dénominations, c'est la manière que Sartre a de qualifier, ou plutôt de disqualifier, sa première philosophie morale qui est intéressante. Il la caractérise en effet comme une morale mystifiée, ou idéaliste ${ }^{6}$. Nous examinerons pourquoi Sartre porte sur sa première philosophie pratique un jugement si sévère et nous chercherons à déterminer si ce jugement est fondé.

Sartre qualifie la deuxième phase de sa pensée morale de « réalisme » et il la situe, dans son entretien avec Michel Sicard, dans les années 1963-1964. Il préparait alors les conférences qu'il voulait présenter dans des universités américaines, réunies sous le titre de Recherches pour une morale. De ces conférences, qui n'ont pas été tenues en raison de la guerre du Viêt-Nam, on conserve environ 600 pages manuscrites que Michel Contat espère pouvoir bientôt publier. De la production sartrienne de cette époque, le lecteur n'a donc aujourd'hui à sa disposition que la conférence «Détermination et Liberté » et la Critique de la raison dialectique. Du fait que Sartre a publié cette dernière œuvre lui-même, elle est une des sources les plus importantes pour l'étude de sa pensée à ce moment-là.

La troisième phase est marquée par ce que Sartre élaborait en 1975 sous le titre de travail « Pouvoir et liberté » (1975). Mais, pour accéder à cette phase de la pensée de Sartre, nous n'avons à notre disposition que les entretiens, du reste contestés, avec Benny Lévy ${ }^{7}$ et l'entretien avec Michel Sicard. Malheureusement, ces sources ne nous donnent qu'une image incomplète et peu fiable de ce qu'étaient en matière de philosophie morale les conceptions de Sartre peu avant sa mort. Nous ne traiterons donc pas de cette dernière phase et nous nous concentrerons sur les deux premières.

Sartre développe sa réflexion éthique sur trois niveaux : le niveau de la morale interne, celui de la morale externe, et celui de la morale historique. Au premier niveau, il s'agit uniquement des conditions à remplir pour que, dans la sphère de l'immanence, de la simple attitude, la vie volontaire de l'individu soit moralement bonne. Sur le plan de la morale externe, il s'agit des conditions de validité des normes extérieures, définissant la vie en société des individus. La morale historique, elle, se place dans le domaine de la validité morale du processus historique. Quoique différemment accentués et articulés, les trois niveaux sont présents à toutes les étapes du développement de la philosophie morale de Sartre. Mais on peut dire qu'il y a un déplacement de l'accent et de l'importance : tandis que dans l'Être et le Néant l'accent est mis sur la morale interne, les Cahiers sont déjà marqués par un déplacement vers le niveau social et historique. Ce dernier se trouve finalement au centre des réflexions de Sartre dans la Critique. Ainsi, comme nous le verrons plus tard, les Cahiers et Saint Genet forment une sorte de pont de transition entre les deux.

7 Comme cela ressort des "Perspectives morales" placées à la fin de L'Être et le Néant, Sartre est conscient du fait que, d'une part, on ne peut formuler une philosophie morale sans tenir compte des données ontologiques, mais que, d'autre part, l'ontologie ne suffit pas à elle seule à fonder des normes morales ${ }^{8}$. Par conséquent, il faut qu'interviennent deux étapes à chaque niveau de la réflexion morale sartrienne : une première étape qui sert à définir la structure ontologique du champ d'action et une seconde qui, en s'interrogeant sur les conditions de validité dans ce champ d'action, passe à la réflexion axiologique proprement dite.

La phase de L'Être et le Néant 
Commençons par l'analyse de la première philosophie morale de Sartre. Comment décrit-il la structure de l'action humaine et quelles sont les normes qu'il veut préconiser pour cette action? La thèse fondamentale de l'ontologie sartrienne est que l'homme est libre, ou plus exactement, en termes sartriens, qu'il est condamné à être libre. Cela signifie d'abord que l'homme projette librement les valeurs qui déterminent son action, que ces valeurs ne lui sont pas données par une puissance extérieure, à l'instar de commandements divins, mais qu'il est lui-même leur seul et dernier fondement. Cela signifie ensuite que l'homme peut réviser à volonté ses valeurs, voire effectuer le célèbre renversement des valeurs (voir $E N$, p. 560). Et cela signifie enfin que, devenu conscient de sa liberté et de la responsabilité qui y est attachée, l'homme est angoissé devant cette responsabilité. C'est dans l'angoisse que l'homme comprend que les valeurs qu'il a projetées prétendent à la validité absolue tout en étant ontologiquement relatives, puisqu'elles dépendent de sa liberté.

La contradiction entre l'exigence d'absoluité et la relativité ontologique des valeurs soulève le problème de la possibilité d'un projet privilégié qui échappe à cette contradiction. Avec cette question, nous avons déjà quitté le domaine de l'ontologie et sommes entrés dans celui de la morale. Il ne s'agit plus ici de chercher des lois ontologiques selon lesquelles l'homme est nécessairement projet de soi-même, mais de déterminer le projet absolument valide, ontologiquement toujours contingent, qui évite les implications négatives de ces lois. Quel est ce projet?

10 Dans L'Être et le Néant, Sartre exprime prudemment sa conception, sous forme de question : «Est-il possible, en particulier, qu'elle [la liberté] se prenne elle-même pour valeur en tant que source de toute valeur...? » (EN, p. 722). Selon Sartre, l'homme doit donc comprendre sa liberté comme valeur et essayer de la réaliser en tant que telle. Mais y a-t-il vraiment un sens à se fixer la liberté comme but, alors que l'ontologie sartrienne montre qu'on est condamné à être libre? Mais ce n'est pas, à notre avis, ce que Sartre a voulu dire lorsqu'il a exigé de comprendre la liberté comme but. Il ne s'agit pas de réaliser la liberté, mais d'adopter une nouvelle attitude de l'esprit, un rapport nouveau à une liberté qui a toujours existé.

11 Pour comprendre en quoi consiste cette nouvelle relation positive de l'homme avec luimême, il faut d'abord préciser quelles sont, d'après Sartre, les attitudes négatives face à sa propre liberté. Pour ce faire, revenons sur la théorie sartrienne du projet! Selon Sartre, l'homme se projette comme un être parfait qui est comme il doit être, à qui aucune qualité ne fait défaut et qui ne doit ce qu'il est qu'à lui-même. Si l'homme pouvait réaliser une telle valeur, c'est-à-dire atteindre à une existence divine, il serait ainsi justifié et délivré de son angoisse. Mais, comme le montre Sartre, ceci est exclu pour des raisons de principe. La structure ontologique de l'être humain, son manque d'être (divin) entraîne que l'homme est injustifié et injustifiable, qu'il est ainsi livré à l'angoisse et qu'il est malheureux.

12 Pour échapper à la conscience malheureuse, les hommes peuvent avoir recours à deux expédients qui consistent tous deux, selon Sartre, en une sorte de politique de l'autruche, c'est-à-dire qui visent à leur dissimuler leur situation existentielle. Le premier expédient est la mauvaise foi (voir EN, p. 94 s.). Elle consiste à faire comme si valeurs et normes venaient d'une instance supérieure (Dieu, la nature ou la société) et à refuser ainsi d'assumer la responsabilité des actes qui découlent de ces valeurs. II y a également mauvaise foi lorsque l'on ne s'identifie pas avec son passé, son caractère ou son corps afin d'éviter le blâme qui les vise. La mauvaise foi est donc une attitude de 
distance vis-à-vis de soi-même et de ses propres valeurs, un «n'être pas ce que l'on est », ce qui est le contraire de l'engagement, du " prendre-son-être-sur-soi ».

Sartre critique cette attitude, parce qu'elle nie et fausse la réalité humaine. Comment corriger cette attitude négative? Sartre discute la possibilité d'une correction qui prendrait la forme de la sincérité (voir $E N$, p. 98 s.). On peut assumer ses erreurs et, au lieu de dire : « ce que vous critiquez, ce n'est pas moi », prendre ce que l'on a fait à son propre compte. On peut s'identifier à son caractère, à son passé, à son corps, de telle façon que toute possibilité de changer, de se dépasser, soit niée. Telle serait alors l'attitude de «l'être ce que l'on n'est pas ».

Toutefois, Sartre condamne autant la sincérité que la mauvaise foi. Car, selon son ontologie, l'homme est un être qui tout à la fois n'est pas ce qu'il est (car il se dépasse) et est ce qu'il n'est pas (à savoir son passé, son caractère, son corps). C'est pourquoi la mauvaise foi et la sincérité sont toutes deux erronées. Chacune, mettant l'accent sur un côté de l'existence humaine, refoule l'autre. L'unique attitude face à soi-même qui sache éviter cette unilatéralité est l'authenticité ${ }^{9}$. Celle-ci est la seule à être moralement valide et, partant, l'exigence d'authenticité représente le premier précepte de la morale sartrienne. Mais en quoi cette attitude consiste-t-elle ? Être authentique, c'est accepter consciemment, prendre sur soi et supporter le paradoxe de l'existence humaine et de notre échec. Dans les Cahiers (p. 490), Sartre écrit qu'il s'agit d'une « saisie thématique de la liberté, de la gratuité, de l'injustifiabilité ». En d'autres termes, l'homme ne peut éviter de projeter sa propre perfection, de tendre vers la réalisation de cette valeur, et il serait faux de vouloir sortir de cet engagement. Mais il serait tout aussi faux de prendre trop au sérieux les valeurs et les normes qui en découlent. Comprendre sa liberté comme valeur signifie saisir et accepter que l'on n'est jamais ce que l'on est, que l'on est toujours ce que l'on n'est pas et vivre en conséquence. L'ontologie de Sartre enseigne qu'il est en mon pouvoir d'annuler toutes les valeurs et sa philosophie morale ajoute : « Reconnais-le, assume-le et vis en conséquence ! ${ }^{10}$

Jusqu'ici nous n'avons analysé que les attitudes qu'on peut avoir vis-à-vis de soi-même. Mais la première morale de Sartre ne se borne pas du tout à ce domaine, elle prend également en considération les attitudes vis-à-vis d'autrui. Il nous faut donc aussi tenir compte de l'aspect intersubjectif de la première morale de Sartre. Ici également, comme dans le cas des attitudes face à soi-même, l'ontologie se borne à décrire les attitudes. Il revient ensuite à la morale proprement dite de les critiquer et de prescrire l'attitude jugée bonne.

Sur le plan de l'ontologie (voir la célèbre $3^{\mathrm{e}}$ partie de L'Être et le Néant), Sartre distingue et oppose tout d'abord deux attitudes face à autrui. On peut, d'une part, objectiver l'autre et sa liberté par le regard et essayer ainsi de s'approprier son existence ; on peut aussi se livrer à son regard et à sa liberté, pour s'approprier ainsi sa liberté indirectement, en devenant un objet. De même que, dans l'attitude vis-à-vis de soimême, la mauvaise foi et la sincérité étaient fausses, de même ces deux positions face à autrui sont erronées. En effet, je ne peux ni me transformer en pur objet (mon objectivité est constamment récupérée par ma subjectivité), ni objectiver la liberté d'autrui (elle reste subjectivité). Ce sont donc des tentatives vouées à l'échec. Sartre les résume sous les noms de " sadisme » et « masochisme » (voir $E N$, p. 431 s. et 447 s.).

Dans L'Être et le Néant déjà, Sartre s'interroge sur la possibilité d'une troisième voie, susceptible de surmonter l'alternative du sadisme et du masochisme ${ }^{11}$. Une première réponse positive est donnée dans L'existentialisme est un humanisme ${ }^{12}$. Ici, la philosophie 
morale de Sartre se montre très proche de la conception kantienne. Ainsi, Sartre ne suit pas seulement Kant en déclarant que la morale consiste en ce que «la liberté veut ellemême et la liberté des autres » (EH, p. 85), mais il reprend aussi la pensée kantienne de l'universalisation des maximes de celui qui agit. L'attitude morale exige que l'individu se considère dans son projet comme législateur. Sartre écrit : «l'homme qui s'engage et qui se rend compte qu'il est non seulement celui qu'il choisit d'être, mais encore un législateur choisissant en même temps que soi l'humanité entière, ne saurait échapper au sentiment de sa totale et profonde responsabilité » $(E H$, p. 28$)$. De là résulte directement la formule universaliste de l'impératif catégorique, telle que nous la trouvons chez Kant. Sartre écrit littéralement: "Mais en vérité, on doit toujours se demander : qu'arriverait-il si tout le monde en faisait autant?» (EH, p. 28-29).

Les Cahiers pour une morale

Mais la véritable réponse aux questions morales posées dans L'Être et le Néant ne se trouve pas dans L'existentialisme est un humanisme qui est un texte polémique, prononcé d'abord à l'occasion d'une conférence publique et publié après sans changer le style plutôt oral, mais dans les Cahiers pour une morale ${ }^{13}$. En effet, comme le laisse entendre Arlette Elkaïm-Sartre dans sa préface, ces textes rédigés en 1947 et 1948 et publiés après la mort de Sartre contiennent le développement le plus avancé et le plus articulé de la première morale de Sartre. Cependant, ces textes inachevés et écrits au courant de la plume posent de graves problèmes à l'interprète. Avant d'entamer l'analyse de leur contenu, j'essaierai donc tout d'abord de clarifier leur statut et leur structure.

Pour ce faire, il faut surtout essayer de répondre aux questions suivantes :

20 a) Les deux cahiers forment-ils réellement un ensemble, comme le pense l'éditrice Arlette Elkaïm-Sartre? Sartre les a intitulés « Notes pour une morale, Tome I et Tome II ». Qu'est-ce qui prouve qu'ils sont plus que des notes?

b) Quelle est la relation entre les textes des deux cahiers?

Si on regarde la transition du Tome I au Tome II (p. 426-429), on a l'impression d'une continuité thématique, puisque Sartre parle de l'aliénation à la fin du Tome I et il en parle au début du Tome II. Mais à la page 484, nous trouvons une disposition de l'ouvrage en deux sections qui ne correspond plus à la disposition des textes qui précèdent. La première section est intitulée "Plan d'une morale ontologique", la seconde (p. 487) ne porte pas de titre, mais contient la disposition d'un questionnement sur les relations entre morale et histoire. Or, on s'aperçoit que le premier cahier est consacré, dans sa plus grande partie, à la théorie de l'histoire et que le second contient aussi (dans la partie qui précède le "plan d'une morale ontologique ») des réflexions sur l'histoire et (dans la partie qui suit ce "plan») une partie intitulée «La conversion ", donc le développement de ce qui - selon ce plan - devrait figurer comme paragraphe sept de la première section. Ne faut-il pas abandonner la lecture qui s'impose à première vue, selon laquelle les deux cahiers se suivent selon un plan prémédité, et admettre qu'ils traitent plus ou moins des mêmes sujets, mais selon une disposition différente? Il est intéressant de noter dans ce contexte que le premier cahier contient lui aussi des plans. Le premier (p.15), probablement incomplet, articule, en six paragraphes numérotés, la disposition de la théorie morale, le second (p. 26) esquisse en soixante-huit paragraphes numérotes et en partie rédiges la théorie de l'histoire à laquelle Sartre donne le titre « Ambivalence de l'histoire. Ambiguïté du fait historique ». 
constat ne laisse-t-il pas supposer que les deux cahiers ne contiennent pas une seule théorie en elle-même consistante, mais plutôt plusieurs phases successives du développement de la pensée de Sartre? En effet, il semble que Sartre n'avait pas été satisfait de la disposition qu'il avait suivie jusqu'à la page 484 et qu'il avait l'intention de recommencer son entreprise depuis le début d'après un plan différent pour la laisser plus tard inachevée à son tour.

Si le plan présenté au second cahier contient effectivement la structure définitive de l'ouvrage, quelles sont exactement les parties du texte auxquelles se réfèrent les différents paragraphes de la Section I «Plan d'une morale ontologique » et de la Section II ? Il faut tout d'abord remarquer que l'intitulé "Plan d'une morale ontologique ", placé p. 484 sous le titre "Section I », contient le titre que Sartre voulait donner à la seule section I et non, comme le présume A. Renaut (op. cit., p. 207), un titre que les deux sections auraient en commun. Si cela est exact, la première section embrasse l'ensemble de la «morale ontologique " (et non, comme le pense A. Renaut, la seule théorie de la liberté), alors que la seconde concerne la relation entre la «morale et l'histoire ». De ces deux sections, seul le paragraphe 7 de la Section I est rédigé. Il occupe le reste du second cahier après le plan de la Section II, comme Sartre l'indique explicitement au bas de la p. 487. Cela signifie que l'interprète dispose uniquement des indications sommaires du plan lui-même pour déterminer ce qu'aurait été le contenu des $\$ 8-9$, censés développer la théorie des relations morales entre «mon Pour-soi et mon Pour-autrui » et la signification du règne de la morale. Certes, on peut essayer de combler ces lacunes par des développements analogues dans le premier cahier. Mais, vu la relation entre les deux cahiers, une extrême prudence est de mise dans cette procédure.

25 Après ces remarques sur la structure de l'œuvre, j'aimerais esquisser de manière générale les thèses principales que Sartre y développe. Cependant, aussi longtemps qu'une analyse détaillée et approfondie des Cahiers manquera, les appréciations que je porterai sur la teneur de cet ouvrage resteront provisoires. Chaque lecteur attentif s'aperçoit que les thèses défendues par Sartre dans les Cahiers semblent aller dans des directions opposées, voire se contredire nettement. Nous avons affaire ici d'une part à un humanisme universaliste, à l'instar de l'éthique kantienne, avec la cité des fins comme principe suprême, et d'autre part à l'affirmation que l'impératif catégorique est insuffisant pour faire un choix concret et que la poursuite de la cité des fins serait même interdite si sa réalisation n'était pas impossible d'emblée. Comment résoudre cette aporie?

À mon avis, la résolution de l'aporie se trouve dans l'explication des deux sections que l'éthique de Sartre devait comprendre selon le plan du second cahier. Pourquoi Sartre ne se contente-t-il pas d'une éthique tout court? Pourquoi son éthique se divise-t-elle en une éthique ontologique et une éthique historique ? Pour répondre à ces questions, il faut d'abord clarifier la signification du titre «morale ontologique ». Lorsque Sartre se propose d'élaborer une morale ontologique, il ne veut pas du tout - comme le soutient A. Renaut (op. cit., p. 207) - «déduire de son ontologie une morale ». Cela n'est pas faisable comme Sartre le reconnaît explicitement (L'Être et le Néant, p. 720). Mais comme nous l'avons déjà expliqué plus haut - on ne saurait non plus répondre à la question fondamentale de toute morale: «Que devons-nous faire?" sans avoir déterminé au préalable les structures ontologiques du champ de l'action humaine. Par conséquent, la morale ontologique de Sartre sera une morale qui tient compte des 
rapports à soi-même, à autrui et au monde qui constituent le cadre ontologique de toute action. Nous avons déjà analysé ce que sont ces rapports d'après la théorie de L'Être et le Néant. L'être humain (le Pour-soi) vacille d'une part entre deux attitudes fausses vis-à-vis de soi-même, la mauvaise foi et la sincérité, et d'autre part entre deux attitudes non valables vis-à-vis d'autrui, le sadisme et le masochisme. Cependant, une troisième relation est envisageable qui dépasse les aspects négatifs des deux autres rapports. Or, la tâche que Sartre assigne à son éthique ontologique est d'élaborer ces deux rapports valables (à soi et à autrui) et d'en déterminer la relation.

Quels sont ces deux rapports positifs et valables? Nous avons déjà montré que dans L'Être et le Néant Sartre esquisse la structure d'un rapport valable vis-à-vis de soi-même qu'il appelle " authenticité » et qui consiste en la libre acceptation du fait que je ne suis pas qui je suis et je suis qui je ne suis pas. En ce qui concerne le rapport valable à autrui nous en trouvons les meilleures explications dans certains passages des Cahiers: la solution consiste dans la reconnaissance mutuelle des deux libertés ${ }^{14}$, qui est possible non pas dans une relation directe avec l'autre, mais par l'intermédiaire d'un tiers, qui est la valeur projetée par autrui et le but qu'il vise. Je reconnais la liberté d'autrui non en m'appropriant son but, mais en voulant la réalisation de son but comme étant le sien : «... je ne veux cette fin que tant que l'autre la voudra et que je conçois dans le moment même où je me mets à l'œuvre pour l'aider, que cette fin ne tient que parce qu'il la veut et qu'elle ne doit pas être poursuivie par moi s'il cesse de la vouloir » (Cahiers, p. 291). Il y a donc vis-à-vis d'autrui également une attitude positive. Cette attitude se manifeste par la libre reconnaissance de la liberté d'autrui, par la compréhension, le don de soi, la générosité, l'amour et par l'appel à la liberté d'autrui. C'est ce que veut dire la formule: «comprendre la liberté comme valeur» dans le contexte social. Il y a donc pour Sartre un impératif qui exige la libre reconnaissance mutuelle des libertés.

Dans la partie ontologique de son éthique se trouvent donc les conceptions universalistes, la thèse que la libre reconnaissance de ma propre liberté implique celle de la liberté d'autrui et l'idée d'inspiration kantienne de la cité des fins. Il est possible que lorsqu'il rédigeait L'Être et le Néant, Sartre ait eu l'intention de limiter son éthique à cette «morale ontologique ». Mais déjà dans cet ouvrage, il déclare que la liberté n'est jamais abstraite, mais s'exerce nécessairement dans une situation concrète. D'où la thèse - exprimée dans L'existentialisme est un humanisme - que l'impératif catégorique ne suffit pas, que la morale doit à tout moment être réinventée et concrétisée selon la situation.

Dans les Cahiers, Sartre soutient que la situation concrète ne peut être qu'historique. D'où la nécessité d'un second volet de la théorie morale défini comme éthique historique. Cette seconde éthique critique et relativise la première. En effet, Sartre soutient ici qu'«on peut concevoir formellement le règne des fins mais non matériellement à partir de la situation donnée » (p.487), que l'humanité ne possède qu'une "quasi subjectivité " (on s'étonne qu'A. Renaut ait trouvé cette affirmation étonnante), qu'elle ne saurait s'ériger en vraie totalité, mais restera toujours " totalité détotalisée ». Mais il faut s'entendre sur la signification que Sartre prête à cette formule. Il explique : «Totalité, elle serait à elle-même sa loi dans l'autonomie de la décision libre. Détotalisée entièrement, elle se résoudrait en individus souverains " (Cahiers, p. 283). L'existentialisme serait un simple individualisme si Sartre recommandait cette dernière possibilité comme celle que la morale nous impose de 
poursuivre. Mais, tout au contraire, il définit la vraie morale concrète comme celle qui nous demande de préparer le règne des fins, donc de toujours chercher à dépasser l'individualisme et l'égoïsme des classes vers la « totalisation dans la liberté » (ibid.).

On voit donc facilement comment les contradictions apparentes des affirmations de Sartre peuvent être conciliées. La thèse récurrente des Cahiers et de Saint Genet est que la morale est à la fois nécessaire et impossible. Elle est nécessaire, parce que sans elle l'histoire n'aurait pas de sens (Cahiers, p. 487), elle est impossible parce qu'on ne peut pas être parfaitement moral dans une situation historique immorale. Les thèses qui semblent se contredire appartiennent donc à deux volets différents de la théorie de Sartre. Ce qui est tout à fait vrai au niveau abstrait de l'éthique ontologique peut être faux au niveau concret de la morale historique. Les contradictions disparaissent si on tient compte du niveau théorique auquel les affirmations appartiennent.

31 Voyons maintenant comment Sartre conçoit le passage de l'attitude fausse vis-à-vis de soi-même et vis-à-vis d'autrui à l'attitude juste et valable. Pour décrire ce passage, il emploie le terme chrétien de "conversion » et il en traite dans le paragraphe 7 de la Section I du plan du second cahier. Il dit ceci : « La conversion : réflexion non complice. Motif de la conversion : impossibilité de se récupérer. Sens de la conversion : rejet de l'aliénation» (p. 486) Et Sartre ajoute: "La suppression de l'aliénation doit être universelle. Impossibilité d'être moral seul » (p. 487). Sartre conçoit donc la conversion comme un choix qu'on fait face à autrui et sur la base d'un appel à autrui. Un peu plus loin, Sartre dit ceci : "Et sans doute il existe un universel. Mais il est lui-même vécu historiquement» (p.505). Cela veut dire entre autres que l'universel qu'on utilise pour juger une action ou une théorie est toujours mis en vigueur par des hommes dans une situation historique concrète (voir p. 507). C'est pourquoi la conversion implique une action sociale, une révolution et donne ainsi un sens à l'histoire. «L'histoire implique la morale (sans conversion universelle, pas de sens à l'évolution ou aux révolutions). La morale implique l'Histoire (pas de moralité possible sans action systématique sur la situation)» (p. 487).

Selon A. Renaut (op. cit., p. 229), Sartre reconnaît ici qu'il se trouve face à un cercle vicieux. Pour que le processus historique de la libération puisse être déclenché, il faudrait que les individus se soient déjà arrachés de la réification, mais cela présuppose que la dynamisation historique ait déjà dépassé dans une certaine mesure l'aliénation. À mon avis, tel ne peut être le problème que Sartre pose, car il est convaincu que l'individu n'est jamais totalement livré aux contraintes de la situation. Il s'agit plutôt de savoir comment déterminer le rapport entre l'histoire et la morale, si la morale doit être la médiation entre l'universel et l'histoire. C'est pourquoi Sartre cherche une nouvelle conception de l'histoire, autre que celle de Hegel ou de Marx. A. Renaut a tort de prendre l'affirmation de Sartre : «s'il y a une Histoire c'est celle de Hegel» (Cahiers, p. 31) comme preuve qu'il accepte la théorie hégélienne de l'histoire. Sartre veut dire que si l'histoire forme une unité ou totalité, c'est celle de Hegel. Mais il nie justement qu'elle puisse être une totalité. Selon lui elle restera toujours - comme nous l'avons vu plus haut - une totalité détotalisée.

Voilà donc, grossièrement esquissées, les conceptions morales de Sartre dans la première phase de son développement philosophique. Pourquoi les critique-t-il plus tard en les taxant d'« idéalistes » et de « mystifiantes »?

Saint Genet 

explicitement comme impossible aujourd'hui contribue à la mystification et à l'aliénation des hommes. Le " problème » moral naît de ce que la Morale est pour nous tout en même temps inévitable et impossible. » ${ }^{15}$ Le projet de morale impliquait la liberté sous deux formes : une liberté comme négation de l'existant qui pourrait limiter l'absoluité de la création de soi, et une liberté comme projet positif des possibilités de création de soi. Sartre poursuit en disant que la morale bourgeoise a divisé la liberté en ces deux moments. Elle croit pouvoir retenir le premier, tout en s'abstrayant du second : « L'une [des deux moitiés de la liberté] demeure en nous. Elle identifie pour toujours le Bien à l'Être, donc à ce qui est déjà : l'Être étant la mesure de la perfection, un régime existant est toujours plus parfait qu'un régime qui n'existe pas : on dit qu'il a fait ses preuves ». Il ajoute plus loin : "Tout est plein, tout se tient, tout est en ordre, tout a toujours existé, le monde est un musée dont nous sommes les conservateurs. »

L'autre aspect de la liberté réapparaît sous une forme tout aussi mystifiée: «Substantifiée, séparée de toute intention positive, elle devient négation pure et qui se pose pour soi, pure rage de détruire qui tourne en rond: le Mal. Le mal c'est l'unité de toutes ses impulsions à critiquer, à juger, à rejeter... » (Saint Genet, p. 34-35).

Dans la mesure où la philosophie morale abstraite semble justifier ce processus, elle contribue à maintenir cet état de choses. Elle se transforme en idéologie. Car, dans les faits, la praxis des gens honorables est en partie confirmée par la philosophie morale. Un système de normes déjà existant n'a-t-il pas plus de droits qu'un système simplement potentiel? Pour se réaliser, celui-ci doit tout d'abord prendre la forme de la négation, il doit passer par le stade de la négation du caractère obligatoire des normes positives en vigueur. Le révolutionnaire est solitaire, sa justification est seulement subjective, elle se limite à ce qu'il y a de bon dans son intention. Le défenseur de l'ordre existant, en revanche, est objectivement en son droit, quelle que soit son intention : il se bat contre l'aspect objectif de la liberté révolutionnaire, c'est-àdire contre la destruction pure. Il ne peut prendre connaissance des intentions du révolutionnaire, pas plus qu'il n'est tenu de le faire. Seule une révolution réussie permet d'échapper à ce dilemme, mais pour tomber dans un autre; dans sa réussite, elle acquiert la validité positive, mais elle y perd la validité morale. Seule une révolution avortée est moralement justifiée, mais elle est, sur le plan de l'histoire, un échec.

Sur le plan de la validité, la morale est donc en butte à des contradictions insolubles. L'absoluité de la morale subjective brise la validité positive de la morale objective et celle-ci étouffe l'absoluité du projet dans la contingence. Le moralisme des gens honorables sacrifie l'absoluité à la validité positive, le devoir à l'être. L'amoralisme de la révolution sacrifie la validité positive à l'absoluité, l'être au néant.

La vraie morale que Sartre recherchait dans les Cahiers consisterait dans un dépassement de ces contradictions. Mais il dit à son propos, dans Saint Genet : «Ou la morale est une faribole ou c'est une totalité concrète qui réalise la synthèse du Bien et du Mal. Car le Bien sans le Mal c'est l'Être parménidien, c'est-à-dire la Mort ; et le Mal sans le Bien, c'est le Non-Être pur. On comprendra, j'espère, qu'il ne s'agit nullement d'un "au-delà" nietzschéen du Bien et du Mal, mais plutôt d'une "Aufhebung" hégélienne. La séparation abstraite de ces deux concepts exprime simplement l'aliénation de l'homme. Reste que cette synthèse, dans la situation historique, n'est pas réalisable » (op. cit., 177). 

synthèse est impossible en pratique, c'est-à-dire dans la vie concrète, mais que même une théorie d'une telle praxis, c'est-à-dire une éthique, est impossible. Nous devons donc nous demander quel élément nouveau dans sa pensée a contraint Sartre à abandonner ses anciennes idées, cet abandon marquant le passage de sa première à sa deuxième philosophie morale, que nous allons examiner maintenant.

La phase de la Critique de la raison dialectique niveaux de la théorie: on trouve, d'une part, une théorie descriptive qui analyse la structure de la réalité dans laquelle la morale va être appliquée et, d'autre part, la théorie normative proprement dite. Mais, cette fois, la théorie descriptive ne sera pas une ontologie de la réalité humaine, mais une philosophie de la société et de l'histoire.

Quelles sont alors les structures du champ de la pratique que Sartre considère comme significatives pour sa nouvelle morale ? De manière générale, on peut dire que Sartre, à l'instar de Marx, conçoit l'histoire comme une succession de luttes de classes. À la base de ces luttes, on trouve un fait contingent fondamental qui s'avère être d'une importance capitale pour le problème de la morale: selon Sartre, en effet, la praxis humaine se déroule dès les débuts de l'histoire et jusqu'à nos jours sous le règne de la rareté $^{16}$. Il entend par ce terme la "pénurie des moyens matériels assurant l'existence biologique "; la rareté est donc tout autre chose que le manque dont traitait L'Être et le Néant.

42 C'est en raison de la rareté que dans le domaine social chaque homme devient, du simple fait de son existence, un ennemi potentiel, voire une menace de mort pour tout autre homme. Chacun est en puissance celui qui prive autrui des moyens d'existence nécessaires et chacun est potentiellement " de trop », c'est-à-dire celui que la société ne peut plus nourrir avec les moyens de production disponibles.

On ne saurait surestimer la portée de cette théorie pour la nouvelle conception que Sartre se fait de la morale. Cette théorie introduit en effet une cause extérieure à la décision morale du sujet et à son intention pour expliquer la présence du mal dans le monde. Sartre renverse ainsi le mythe du péché originel : l'homme ne doit pas « gagner son pain à la sueur de son front » parce qu'il a péché, mais c'est parce que la rareté le contraint à " gagner son pain à la sueur de son front ». Il sait que le mal existe dans le monde, sous forme de praxis humaine. Sartre radicalise ainsi également le dicton de Hobbes, homo homini lupus (CRD, I, p. 192). Mais, tandis que, pour ce dernier, l'hostilité entre les hommes peut être surmontée par la création de l'État, chez Sartre, c'est l'histoire, interprétée de façon matérialiste, qui résoudra l'antagonisme entre les hommes en supprimant progressivement la rareté.

Dans ce contexte, Sartre rappelle qu'à son premier stade, l'éthique se développe comme un manichéisme. Le mal et le bien sont des constituants objectifs du monde. Ce manichéisme prend les formes historiques les plus diverses. La plus importante est la société de classes, qui repose sur le fait que les conditions économiques de la lutte contre la rareté, à savoir ce que Marx nomme les forces de production, exigent une certaine forme d'organisation de la société. Ainsi, les conditions capitalistes de cette lutte exigent une classe de capitalistes et une classe de travailleurs. Partant, le mal objectif apparaît sous la forme de l'ennemi de classe. 

Considérons tout d'abord la morale de la classe dominante. Sartre dit à son propos, dans « Détermination et liberté ${ }^{17}:$ « ...les bourgeois, par exemple, sont les produits du système capitaliste, mais ils le maintiennent et le perpétuent sans arrêt, non par inertie, mais par choix, à travers l'élaboration d'une stratégie économique, sociale et politique»(p. 744). Cela signifie que les valeurs dont les bourgeois se servent pour perpétuer le système capitaliste leur semblent être d'une validité absolue, être obligatoires pour toute l'humanité. Par exemple, l'humanisme bourgeois considère le développement et l'épanouissement de toutes les facultés humaines dans les sciences, l'esprit, l'art et la culture comme le but suprême de la réalisation personnelle, c'est-àdire comme le bien tout court. Seul l'homme cultivé est un être à part entière ; l'inculte, le prolétaire, apparait ainsi objectivement comme un sous-homme. Puisque ce dernier ne s'est pas façonné comme homme, il semble juste de le traiter comme une chose, de l'exploiter comme une bête de somme. Selon Sartre l'humanisme présente donc deux faces contradictoires : d'une part, il justifie l'exploitation capitaliste, mais, d'autre part, il en exige la suppression, car il définit les valeurs humanistes comme valables pour tous, exigeant ainsi l'émancipation du travailleur et son passage à la condition d'homme. bourgeoise, sont les premiers à en éprouver la contradiction comme une provocation. En même temps qu'ils fournissent une justification à la classe bourgeoise, ils en deviennent la mauvaise conscience. Toute leur activité consiste à essayer d'échapper à la complicité dans l'exploitation, mais c'est une tentative vaine. Ils nient la classe bourgeoise et ses valeurs, ils transgressent les bonnes mœurs, le droit et la morale, mais ils n'en retirent pas de justification, puisqu'ils demeurent, malgré tout, bourgeois. Selon Socrate, c'est en souffrant l'injustice qu'on est justifié. Dans cette optique, la justification est réservée à l'exploité et non à l'exploiteur. La tentative illusoire des intellectuels bourgeois de s'intégrer à la classe des exploités prouve une fois de plus que dans la société de classes le bien et le mal sont des phénomènes objectifs sur lesquels le processus personnel de décision n'a pas d'influence. D'un point de vue moral, la protestation intellectuelle est bien sûr infiniment supérieure à l'autosatisfaction repue des petit-bourgeois, mais ceci ne change rien au fait qu'elle reste enchaînée aux conditions de la classe bourgeoise.

Qu'en est-il pour Sartre de la morale de la classe ouvrière ? Elle est également ambiguë et contradictoire, mais dans un tout autre sens. La classe des travailleurs est le produit du système capitaliste, elle en est le moment nécessaire. La morale des prolétaires n'est rien d'autre qu'une morale bourgeoise, puisque les valeurs existant à l'intérieur du système sont dictées par la classe dominante. Cependant, à la différence du bourgeois, le prolétaire ne peut jamais se flatter de satisfaire aux exigences de ces valeurs. C'est en adhérant à elles que le prolétaire se condamne à être le sous-homme. Cependant, comme le souligne Sartre, il ne peut, à l'intérieur du système capitaliste, qu'y adhérer : « la nécessité de vivre les contraint [les classes exploitées] à accepter les règles du jeu » (Détermination, p. 744).

48

Le travailleur ressent que la morale bourgeoise lui est hostile, qu'elle le diminue et le définit comme le mal, et, en même temps, l'expérience lui enseigne qu'il peut au mieux y échapper en tant qu'individu, mais pas en tant que membre de sa classe. Surmonter le mal, c'est donc pour la classe ouvrière dépasser le système capitaliste. Le premier pas 
dans ce sens est le refus de reconnaître la morale bourgeoise, ce qui ne peut se faire de façon abstraite et théorique, mais doit s'inscrire dans une dialectique historique : « en refusant l'humanisme en tant que tel, l'ouvrier avouerait qu'il est non humain; l'exigence nouvelle, née du dépassement de cette contradiction, c'est que le refus soit inscrit dans la production d'un humanisme vrai et positif » (CRD, I, p. 740-741).

Dans la perspective de la philosophie sociale de Sartre, toute morale positive est donc irrémédiablement ambiguë. Sartre l'explique dans les termes suivants : «Ce qui fait l'ambiguïté de toute morale passée et de toute morale actuelle, c'est que la liberté comme relation humaine se découvre elle-même, dans le monde de l'exploitation et de l'oppression, contre ce monde et comme négation de l'inhumain à travers les valeurs mais qu'elle s'y découvre aliénée et qu'elle s'y perd et que, par les valeurs, elle réalise malgré tout l'exigence indépassable que l'être pratico-inerte lui impose [...]. Tout système de valeurs repose sur l'exploitation et l'oppression [...]; tout système de valeurs confirme l'exploitation et l'oppression [...]; tout système de valeurs, en tant qu'il est soutenu par une pratique sociale, contribue directement ou indirectement à mettre en place des dispositifs et des appareils qui, le moment venu [...] permettront de nier cette oppression et cette exploitation... » (CRD, I, p. 302-303).

Que signifie cette théorie de la société et de l'histoire quant à la possibilité d'une philosophie morale normative? Celle-ci est-elle une fois pour toutes réduite à l'absurde, ou Sartre maintient-il l'idée d'un projet absolument valable de praxis humaine également au deuxième stade de sa philosophie morale ? À ce sujet, deux remarques s'imposent :

51 1. Dans la situation historique concrète, qui est celle de la rareté, il s'agit tout d'abord d'améliorer les conditions d'existence, c'est-à-dire de vaincre la rareté. C'est dans ce sens qu'il faut comprendre ce que Sartre écrit dans la Critique de la raison dialectique, avec une pointe contre Kant: "N'allons pas croire, en effet, que nous sommes entrés dans la cité des fins et que, dans la réciprocité, chacun reconnaît et traite l'Autre comme fin absolue. [...] Cette hypothèse même nous renverrait à l'idéalisme absolu : seule une idée au milieu d'autres idées peut se poser comme sa propre fin. Mais l'homme est un être matériel au milieu d'un monde matériel; il veut changer le monde qui l'écrase, c'est-à-dire agir par la matière sur l'ordre de la matérialité : donc se changer lui-même » (CRD, I, p. 191).

Ceci explique pourquoi, dans sa deuxième phase, Sartre caractérise sa morale comme réaliste. La théorie morale est niée par les résultats de la philosophie sociale et de la philosophie de l'histoire, elle y est absorbée. On pourrait même dire que pour Sartre la théorie morale s'épuise en critique de la morale. Le sens de la philosophie morale paraît uniquement consister dans la constatation de sa propre absurdité.

53 2. Mais cette réponse négative n'est pas tout ce que l'on peut dire à ce sujet. Car Sartre ne se limite pas à critiquer la morale passée et présente. Il définit également les conditions de réussite d'une morale véritable. Le mal objectif disparaittra de l'histoire avec la fin de la rareté. Le bien et le mal deviendront alors de véritables grandeurs morales, dans la mesure où ils prendront leur source exclusivement dans la liberté du sujet et ne seront définis que par les lois de cette liberté. Dans ce sens, Sartre écrit dans la Critique de la raison dialectique: "il doit y avoir une liquidation possible de ces structures [de la rareté et de la structure de classes qui s'ensuit], les valeurs disparaîtront avec elles pour redécouvrir la praxis dans son libre développement, 
comme seule relation éthique de l'homme avec l'homme en tant qu'ils dominent cependant la matière » (CRD, I, p. 302).

Cependant, on trouve dans la philosophie de Sartre un reste d'idéalisme, un noyau idéaliste qui résiste à toutes les conversions réalistes. Ce noyau consiste dans la conviction que la morale est l'idée dominante vers laquelle l'humanité doit se dépasser de système en système. La morale est le sens de l'histoire comme histoire de l'émancipation, mais en tant que telle, elle est au-delà de l'histoire. L'éthique prend ainsi la forme d'une téléologie de l'histoire.

Nous retrouvons ici la structure qui, au niveau de L'Être et le Néant, était caractéristique du mode d'existence du Pour-soi. De même que le constant "devoir-se-dépasser " garantit un sens à l'existence individuelle, de même la révolution permanente des systèmes sociaux donne un sens à l'histoire de l'humanité.

À notre avis, Sartre ne pouvait penser différemment, car il n'y avait rien qui lui inspirât une plus grande horreur que l'immobilité de la vie individuelle ou de l'histoire. La tension vers l'avenir et le progrès ne doivent jamais connaitre de fin et ce sont des idées infinies (au sens de l'idéalisme allemand) qui garantissent que ce dépassement de soi n'aura jamais de fin.

Cet article reprend, complète et actualise la postface de mon livre La Dialectique de Sartre, Lausanne, L'Âge d'homme, 1995. Je remercie l'éditeur d'avoir accepté la republication de ce texte. Une première version de ma reconstruction de la morale de Sartre a été présentée - sous le titre "Wie hätte Sartres Moralphilosophie ausgesehen ? »- au Congrès Sartre tenu à Francfort sur le Main en juillet 1987 et dont les Actes ont été édités par Tr. König sous le titre Sartre. Ein Kongress (Reinbeck, Rowohlt, 1988, p. 276-293).

\section{NOTES}

1.. Mis à part les nombreux passages à ce sujet dans les deux ouvrages principaux, L'Être el le Néant et la Critique de la raison dialectique, ainsi que dans L'existentialisme est un humanisme, Saint Genet, L'Idiot de la famille et d'autres écrits, nous ne disposons, pour reconstituer la philosophie pratique de Sartre, que des Cahiers pour une morale, publiés en 1983 par Arlette Elkaïm-Sartre, mais rédigés en 1947 et 1948 et de la conférence « Détermination et liberté » (1964).

2.. A. RENAUT, Sartre, le dernier philosophe, Paris, Grasset, « Le Collège de philosophie », 1993, p. 241.

3.. Voir G. SEEL, La Dialectique de Sartre, op. cit.

4.. Sartre. Un film, Paris, Gallimard, 1977.

5.. « Gespräch zwischen Jean-Paul Sartre und Michel Sicard », Die Tageszeitung, 11.1.1980.

6.. Voir Obliques 15.

7.. Jean-Paul SARTRE, Benny LÉVY, L'Espoir maintenant : les entretiens de 1980, présentés et suivis du Mot de la fin par Benny Lévy, Paris, Verdier, 1991. 
8.. Jean-Paul SARTRE, L'Être et le Néant. Essai d'ontologie phénoménologique, Paris, Gallimard, « Bibliothèque des Idées », 1943 (les citations vont à la réimpression de 1965), p. 720 : «L'ontologie ne saurait formuler elle-même des prescriptions morales ». On ne peut donc pas dire, comme le fait A. RENAUT, que Sartre tente de « déduire de son ontologie une morale » (op. cit., p. 248).

9.. EN, 111 note (1) : « S'il est indifférent d'être de bonne ou de mauvaise foi [...], cela ne veut pas dire qu'on ne puisse échapper radicalement à la mauvaise foi. Mais cela suppose une reprise de l'être pourri par lui-même que nous nommerons authenticité [n. s.] et dont la description n'a pas place ici ». Nous verrons que Sartre, en utilisant le terme heideggérien « authenticité », vise tout autre chose que Heidegger.

10.. Il est tout à fait significatif dans ce contexte que Sartre présente le jeu, activité non sérieuse par excellence, comme modèle de l'attitude vis-à-vis de soi-même qui échappe à la mauvaise foi. Il écrit que l'étude de ce comportement, qui repose sur la « réflexion purifiante », est l'affaire de l'éthique (voir $E N$, p. 670).

11.. Voir $E N$, p. 484 notes (1), où Sartre dit que « ces considérations [à propos du sadisme et du masochisme] n'excluent pas la possibilité d'une morale de la délivrance et du salut ».

12. Jean-Paul SARTRE, L'existentialisme est un humanisme, Paris, Nagel, « Pensées », 1946, [réimpression 1970]. Les références vont à cette dernière édition.

13.. Jean-Paul SARTRE, Cahiers pour une morale, éd. et intr. par A. El-Kaïm Sartre, Paris, Gallimard, « Bibliothèque de philosophie », 1983.

14.. Cahiers, p. 17 : « Dans le règne des fins : pas de problème. Car si des libertés se voulant libres reconnaissent mon acte comme issu de ma liberté et le reprennent en liberté, je veux à la fois mon acte avec ma liberté et la leur ».

15.. Saint Genet, comédien et martyr (tome I des Euvres complètes de Jean Genet), Paris, Gallimard, 1952, p. 177.

16.. Jean-Paul SARTRE, Critique de la raison dialectique. Tome 1, Théorie des ensembles pratiques, Paris, Gallimard, « Bibliothèque des Idées ", " Rareté et mode de production », 1960 , p. 200 s. Noté $C R D$, I dans la suite.

17.. Jean-Paul SARTRE, « Détermination et liberté », in Michel conTAT et Michel RYBALKA, Les Écrits de Sartre. Chronologie et bibliographie commentée, Paris, Gallimard, 1970, p. 735-745. Cité Détermination dans la suite).

\section{RÉSUMÉS}

Comme Sartre l'a lui-même souligné, il convient de distinguer trois phases dans le développement de sa philosophie morale. La première est théorique, c'est celle de L'Être et le Néant et des Cahiers pour une morale. La seconde correspond à la Critique de la raison dialectique et à Détermination et liberté. Quant à la dernière, dont les grandes lignes sont parues dans les conversations avec Michel Sicard et Benny Levy, elle fut initiée en 1975 sous le titre de travail "Pouvoir et liberté». Comme les affirmations de ces deux auteurs sont toutefois sujettes à caution, nous concentrerons notre attention sur les deux premières phases. Sartre y développe sa théorie morale sur trois niveaux : celui de l'intention, celui des interactions sociales et celui de 
l'histoire. Bien que ces trois niveaux soient tous présents dans les deux premières phases que nous avons distinguées, Sartre attache plus d'attention au premier et au deuxième niveau dans la première phase, alors que dans la dernière, la dimension historique domine clairement sa pensée. Dans la première phase, Sartre développe une théorie morale proche de celle de Kant, en établissant l'idéal du « royaume des fins » comme principe suprême. Il infléchit toutefois dans un sens historique la théorie kantienne en affirmant que cet idéal n'est pas réalisable, et que le « royaume des fins » consiste précisément en la préparation du royaume des fins. La découverte décisive de la seconde phase est l'intuition qu'en raison du manque chronique de moyens d'existence, tout homme est un adversaire pour les autres hommes et une menace permanence pour leurs existences. La question du bien moral n'est plus celle de la bonne volonté et de l'action humaine, mais celle du dépassement de cette situation de rareté. Cette bataille doit cependant être gagnée sur deux fronts: celui du progrès technologique et économique, de manière à pouvoir contrôler les conditions matérielles de l'existence humaine, et celui du combat en faveur des opprimés et des classes exploitées, pour leur libération. La deuxième philosophie morale de Sartre est donc plus d'inspiration marxiste, tandis que la première était inspirée par Kant.

In the development of Sartre's philosophy of morals, we have to distinguish three periods, as Sartre himself emphasized. The first of which is the theory we find in L'Être et le Néant and in the Cahiers pour une morale. The second corresponds to the Critique de la raison dialectique and Détermination et liberté. The last was initiated in 1975 under the working title "Pouvoir et liberté", which outline appears in the conversations with Benny Levy and Michel Sicard. As the latter are not very trustworthy we will concentrate our reconstruction on the first two steps.

Sartre develops his moral theory in three levels: the level of intention, the level of social interaction and the level of history. Though all three levels are present in the first two periods we distinguished, in the first period Sartre pays more attention to the first and second level, while in the last period an historic dimension clearly dominates his thought. In the first period Sartre develops a moral theory in which he comes close to Kant's position, establishing as the highest principle the ideal of the 'realm of ends'. However, he gives this conception a historical twist by saying that the 'realm of ends' can never be reached and that 'the 'realm of ends' precisely consists in the preparation of the 'realm of ends"'. The decisive discovery of the second period was the insight that, due to the scarcity of supplies for living, man is the adversary of other men and a permanent threat to their existence. Therefore the question of moral good is no longer a question of good will and human behaviour, but a question of overcoming the situation of scarcity. However, this is a battle on two fronts : on technological and economic progress, to control the material conditions of human existence, and on the fight for the oppressed and exploited classes for their liberation. So the second moral philosophy follows more Marxist lines, while the first was inspired by Kant.

\section{AUTEUR}

\section{GERHARD SEEL}

Gehrard Seel est Professeur ordinaire de philosophie à l'Université de Berne et Directeur de l'Institut de philosophie à cette université. Il est secrétaire général de l'Académie Internationale de Philosophie de l'Art et éditeur de plusieurs séries philosophiques. Ses principales publications sont : Die Aristotelische Modaltheorie, 1982, La Dialectique de Sartre, 1995 et Ammonius and the Sea-battle, 2001. Il a publié de nombreux articles sur l'histoire de la philosophie, la philosophie pratique, l'esthétique et la philosophie du langage. 